\title{
On the eigenfunction expansion method for semilinear dissipative equations in bounded domains and the Kuramoto-Sivashinsky equation in a ball
}

\author{
by \\ V. V. VArlamov (Austin, TX)
}

\begin{abstract}
Presented herein is a method of constructing solutions of semilinear dissipative evolution equations in bounded domains. For small initial data this approach permits one to represent the solution in the form of an eigenfunction expansion series and to calculate the higher-order long-time asymptotics. It is applied to the spatially 3D Kuramoto-Sivashinsky equation in the unit ball $B$ in the linearly stable case. A global-in-time mild solution is constructed in the space $C^{0}\left([0, \infty), H_{0}^{s}(B)\right), s<2$, and the uniqueness is proved for $-1+\varepsilon \leq s<2$, where $\varepsilon>0$ is small. The second-order long-time asymptotics is calculated.
\end{abstract}

1. Introduction. We are concerned with studying solutions of semilinear dissipative evolution equations in bounded domains. It is well known that the Galerkin method (see, e.g., $[2,20]$ ) permits one to establish existence, uniqueness, and regularity results for such problems. However, this approach does not allow to construct solutions. The use of invariant manifolds for parabolic semilinear equations in bounded domains permits one to prove stability and find the lower-order long-time asymptotics (see $[2,8,37]$ and the references therein). Since the linear operator of the equation has a point spectrum, one can separate the phase space into stable, unstable, and central manifolds. The solutions enjoy exponential decay in time, while the power-law decay is typical for Cauchy problems. As regards the latter, C. E. Wayne [39] examined them for the nonlinear heat equation with a sufficiently smooth nonlinear term, constructed the invariant manifolds, and showed how they can be used to obtain long-time asymptotics.

Our main goal consists, first, in constructing solutions of semilinear dissipative equations governing wave propagation, and second, in obtaining the higher-order long-time asymptotics. The basic ideas of our method were developed in the papers [30-36], and in a certain sense they represent a further development of the approach of [17], where spatially 1D Cauchy problems

2000 Mathematics Subject Classification: 35K55, 35K60, 35B40. 
for the first-order (in time) nonlocal dissipative equations were considered. We begin with the general description of the method and then show its application to the spatially 3D Kuramoto-Sivashinsky (K-S) equation in a ball.

Let $H$ be a Hilbert space with the scalar product $\langle\cdot, \cdot\rangle$ and the corresponding norm $\|\cdot\|$, and let the operator $A$ be defined on $D(A)$ dense in $H$. Assume that $A$ is closed and has a complete orthogonal system $\left\{e_{j}\right\}_{j=1}^{\infty}$ of eigenvectors, $e_{j} \in D(A)$,

$$
A e_{j}=\Lambda_{j} e_{j}, \quad \operatorname{Re} \Lambda_{j}>0, \quad j \in \mathbb{N},
$$

where the eigenvalues $\Lambda_{j}$ are numbered in increasing order of $\operatorname{Re} \Lambda_{j}$, i.e., $\operatorname{Re} \Lambda_{i} \leq \operatorname{Re} \Lambda_{j}$ for $i \leq j$. Moreover, assume that $\operatorname{Re} \Lambda_{j} \rightarrow \infty$ as $j \rightarrow \infty$. We do not suppose that the vectors $e_{j}$ are normalized.

Let $H^{2}$ be the space $\left\{u=\sum_{i=1}^{\infty} \widehat{u}_{i} e_{i}: \sum_{i=1}^{\infty}\left|\widehat{u}_{i}\right|^{2}\left|\Lambda_{i}\right|^{2}\left\|e_{i}\right\|^{2}<\infty\right\}$ endowed with the norm

$$
\|u\|_{2}^{2}=\sum_{i=1}^{\infty}\left|\widehat{u}_{i}\right|^{2}\left|\Lambda_{i}\right|^{2}\left\|e_{i}\right\|^{2}, \quad \widehat{u}_{i}=\frac{\left\langle u, e_{i}\right\rangle}{\left\|e_{i}\right\|^{2}} .
$$

Then the operator $A: D(A) \rightarrow H$ can be extended to a continuous operator from $H^{2}$ into $H$. We will retain the same notation for this operator. Denote by $H^{s}$ the space

$$
H^{s}=\left\{u \in H:\|u\|_{s}^{2}=\sum_{i=1}^{\infty}\left|\widehat{u}_{i}\right|^{2}\left|\Lambda_{i}\right|^{s}\left\|e_{i}\right\|^{2}<\infty\right\} .
$$

Consider the following Cauchy problem:

$$
\begin{aligned}
u^{\prime}(t)+A u(t) & =B(u(t), u(t)), \quad t>0, \\
u(0) & =\phi,
\end{aligned}
$$

where $u(t):[0, \infty) \rightarrow H$ is a continuous function and $B(\cdot, \cdot)$ is a bilinear form. The solution of the corresponding linear problem exists for all $\phi \in H$ and is given by the formula

$$
u(t)=\exp (-t A) \phi=\sum_{i=1}^{\infty} \widehat{u}_{i} \exp \left(-\Lambda_{i} t\right) e_{i},
$$

where $\exp (-t A)$ is a strongly continuous semigroup.

We integrate (1.2) with respect to $t$ and reduce it to the integral equation

$$
u(t)=\exp (-t A) \phi+\int_{0}^{t} \exp (-(t-\tau) A) B(u(\tau), u(\tau)) d \tau .
$$

The function $u(t)$ is called a strong solution of the problem (1.2) if each term in (1.2) is a continuous $H$-valued function of $t$. The function $u(t)$ 
is called a mild solution of (1.2) if it satisfies (1.3) in the Banach space $C^{0}\left([0, \infty), H^{s}\right)$.

We seek solutions of (1.2) in the form

$$
u(t)=\sum_{m=1}^{\infty} \widehat{u}_{m}(t) e_{m}, \quad \widehat{u}_{m}(t)=\frac{\left\langle u(t), e_{m}\right\rangle}{\left\|e_{m}\right\|^{2}} .
$$

We expand the nonlinear term into the series

$$
B(u(t), u(t))=\sum_{m=1}^{\infty} \widehat{B}_{m}(t) e_{m}
$$

where the coefficients $\widehat{B}_{m}(t)$ are calculated in the following way:

$$
\begin{aligned}
\widehat{B}_{m}(t) & =(B(u(t), u(t)))_{m}^{\wedge}(t) \\
& =\frac{\left\langle B\left(\sum_{p=1}^{\infty} \widehat{u}_{p}(t) e_{p}, \sum_{k=1}^{\infty} \widehat{u}_{k}(t) e_{k}\right), e_{m}\right\rangle}{\left\|e_{m}\right\|^{2}} \\
& =\sum_{p, k=1}^{\infty} b(m, p, k) \widehat{u}_{p}(t) \widehat{u}_{k}(t)
\end{aligned}
$$

where

$$
b(m, p, k)=\frac{\left\langle B\left(e_{p}, e_{k}\right), e_{m}\right\rangle}{\left\|e_{m}\right\|^{2}} .
$$

Then we substitute (1.4)-(1.6) into (1.2) and after integrating the result in $t$ obtain

$$
\sum_{m=1}^{\infty} \widehat{u}_{m}(t) e_{m}=\sum_{m=1}^{\infty} \exp \left(-\Lambda_{m} t\right) \widehat{\phi}_{m} e_{m}+\sum_{m=1}^{\infty}\left(\int_{0}^{t} \exp \left(-\Lambda_{m}(t-\tau)\right) \widehat{B}_{m}(\tau) d \tau\right) e_{m}
$$

which implies that

$$
\begin{aligned}
\widehat{u}_{m}(t)= & \exp \left(-\Lambda_{m} t\right) \widehat{\phi}_{m} \\
& +\int_{0}^{t} \exp \left(-\Lambda_{m}(t-\tau)\right) \sum_{p, k=1}^{\infty} b(m, p, k) \widehat{u}_{p}(\tau) \widehat{u}_{k}(\tau) d \tau, \quad m \in \mathbb{N} .
\end{aligned}
$$

Next, we assume that the initial data in (1.2) is small and set $\phi=\varepsilon \widetilde{\phi}$, where $\varepsilon=\|\phi\|, \widetilde{\phi}=\phi /\|\phi\|$. Replacing $\widehat{\phi}_{m}$ in (1.7) by $\varepsilon \widetilde{\phi}_{m}$ we seek $\widehat{u}_{m}(t)$ in the form of the series in $\varepsilon$

$$
\widehat{u}_{m}(t)=\sum_{N=0}^{\infty} \varepsilon^{N+1} \widehat{v}_{m}^{(N)}(t)
$$

Substituting (1.8) into (1.7) we get the following recurrence formulas: 


$$
\begin{aligned}
\widehat{v}_{m}^{(0)}(t)= & \exp \left(-\Lambda_{m} t\right) \widetilde{\phi}_{m}, \\
\widehat{v}_{m}^{(N)}(t)= & \int_{0}^{t} \exp \left(-\Lambda_{m}(t-\tau)\right) \\
& \times \sum_{p, k=1}^{\infty} b(m, p, k) \sum_{j=1}^{N} \widehat{v}_{p}^{(j-1)}(\tau) \widehat{v}_{k}^{(N-j)}(\tau) d \tau .
\end{aligned}
$$

Thus, the formal solution of (1.2) with small initial data is presented in the form $(1.4),(1.8),(1.9)$. In order to justify these considerations we should secure the convergence of the series in (1.4), (1.6), (1.8), (1.9). To this end we establish the following estimates for integers $N \geq 0, m \geq 1$, and $t \geq 0$ :

$$
\left|\widehat{v}_{m}^{(N)}(t)\right| \leq c^{N} f_{1}(N) f_{2}(m) \exp \left(-\Re_{m} t\right),
$$

where $æ_{m}>0$, the constant $c>0$ is independent of $N, m, t ; f_{1}(N)$ tends to zero sufficiently fast to guarantee the absolute convergence of

$$
\sum_{j=1}^{N} \widehat{v}_{p}^{(j-1)}(t) \widehat{v}_{k}^{(N-j)}(t) \quad \text { as } N \rightarrow \infty,
$$

and the decay of $f_{2}(m)$ is sufficient for the absolute convergence, uniform in $m \geq 1, t \geq 0$, of the series

$$
\sum_{p, k=1}^{\infty} b(p, k, m) \sum_{j=1}^{N} \widehat{v}_{p}^{(j-1)}(t) \widehat{v}_{k}^{(N-j)}(t) .
$$

The inequalities (1.10) are established by induction, and the behavior of the zero iteration $\widehat{v}_{m}^{(0)}(t)$ serves as a starting point.

Next, we choose $\varepsilon \in\left[0, \varepsilon_{0}\right]$, with $\varepsilon_{0}<1 / c$, so that the series (1.8) converges uniformly with respect to $t \geq 0, \varepsilon \in\left[0, \varepsilon_{0}\right]$. The estimate of $\widehat{u}_{m}(t)$ deduced on the basis of (1.8), (1.10) permits one to establish that each term in the equation (1.2) is a continuous $H$-valued function of $t$ in the case of strong solutions or that $u(t):[0, \infty) \rightarrow H^{s}$ is continuous in the case of mild solutions.

Up to this point we have not used to the full extent the dissipativity of the equation, and the method described above can be used for constructing solutions of conservative equations as well (at least for a bounded time interval, and sometimes for all $t \geq 0$ ). The exponential decay in (1.10) will be essentially used to obtain the higher-order long-time asymptotics, but we would like to illustrate it by a concrete example: the Kuramoto-Sivashinsky equation in a ball. An application of the method to second-order (in time) evolution equations in one and two space dimensions can be found in [30-34]. The nonlinear fractional Laplacian heat equation in 2D and 3D was studied in $[35,36]$. 
In principle, we can also treat a power nonlinearity in $(1.2)$, i.e., $(u(t))^{n}$ with an integer $n \geq 3$. We can expand it into the eigenfunction series

$$
(u(t))^{n}=\sum_{m=1}^{\infty}\left(u^{n}\right)_{m}^{\wedge}(t) e_{m}
$$

where

$$
\begin{aligned}
& \left(u^{n}\right)_{m}^{\wedge}(t) \\
& =\frac{\left\langle\sum_{p_{1}=1}^{\infty} \widehat{u}_{p_{1}}(t) e_{p_{1}} \cdot \sum_{p_{2}=1}^{\infty} \widehat{u}_{p_{2}}(t) e_{p_{2}} \ldots \sum_{p_{n}=1}^{\infty} \widehat{u}_{p_{n}}(t) e_{p_{n}}, e_{m}\right\rangle}{\left\|e_{m}\right\|^{2}} \\
& \quad=\sum_{p_{1}, \ldots, p_{n} \geq 1} b\left(m, p_{1}, \ldots, p_{n}\right) \widehat{u}_{p_{1}}(t) \ldots \widehat{u}_{p_{n}}(t)
\end{aligned}
$$

where

$$
b\left(m, p_{1}, \ldots, p_{n}\right)=\frac{\left\langle e_{p_{1}} \ldots e_{p_{n}}, e_{m}\right\rangle}{\left\|e_{m}\right\|^{2}},
$$

and apply the same procedure as above. Our main concern will be the convergence of the series in (1.11) and much will depend on obtaining subtle estimates of the coefficients $b\left(m, p_{1}, \ldots, p_{n}\right)$.

Below we shall consider the K-S equation

$$
\partial_{t} u+\nu \Delta^{2} u+\Delta u=|\nabla u|^{2},
$$

where $u=u\left(x_{1}, x_{2}, x_{3}\right), \nu=$ const $>0, \nabla u=\operatorname{grad} u$, and $\Delta$ is the Laplace operator in $x_{1}, x_{2}, x_{3}$. This equation arises in the theory of long waves in thin films [3, 29], of long waves at an interface between two viscous liquids [6], in systems of reaction-diffusion type [11, 12], and in the description of the nonlinear evolution of a linearly unstable flame front [24, 25]. The linear terms in (1.12) describe the interaction of long-wavelength pumping and short-wavelength dissipation, and the nonlinear term characterizes energy redistribution between various modes.

The K-S equation was extensively studied in the eighties (mostly in the spatially 1D case), both in the context of inertial manifolds and in numerical simulation of dynamical behavior (see $[1,5,6,13,18,19]$ ). In $[14,15]$ Michelson showed that a slight modification of the spatially $2 \mathrm{D}$ equation $(1.12)$,

$$
\partial_{t} u+\nu \Delta^{2} u+\Delta u+|\nabla u|^{2}=c^{2},
$$

has stationary solutions. In the context of combustion theory these solutions represent Bunsen flames on infinite linear or circular burners. Examining spatially periodic solutions, Nicolaenko, Scheurer, and Temam [19] showed that the existence of a global absorbing ball implies the existence of a global attractor, and gave an upper estimate of its Hausdorff dimension. Under the assumption that the initial data is odd, they proved the existence of a global 
absorbing set in $L_{2}(0, l)$ for the derivative K-S equation. Collet et al. [4] and independently Goodman [7] got rid of this antisymmetry requirement.

In the spatially $2 \mathrm{D}$ case an important problem was to show the existence of a bounded absorbing set in $L_{2}(\Omega)$. Using the method of [21], Sell and Taboada [23] gave the answer to this question via proving the existence of a bounded local absorbing set in $H_{\text {per }}^{1}([0,2 \pi] \times[0,2 \pi \varepsilon])$ for sufficiently small $\varepsilon$. Molinet [16] improved their results and gave sufficient conditions for the local stability of the solutions of the derivative K-S equation with spatially periodic boundary conditions in a thin rectangular domain.

We shall examine the first initial-boundary problem for the 3D K-S equation in a ball with small initial conditions and homogeneous boundary conditions. For the linearly stable case $\nu>1 / \Lambda_{1}$ we shall construct its mild global-in-time solutions in the form of an eigenfunction expansion. The uniqueness will follow from the analysis of the corresponding nonlinear integral equation. Then we shall calculate the second-order long-time asymptotics of the solution in question. The nonlinear stability result for the case $0 \leq \nu \leq 1 / \Lambda_{1}$ (but not the asymptotics) can be obtained by the considerations analogous to those of [23]. However, to calculate the long-time asymptotic expansion we need the linear stability of the equation.

2. Notation and function spaces. We denote by $B$ the ball of unit radius and introduce the coordinate system with origin at the center of the ball, so that $B=\{(r, \theta, \varphi):|r|<1,0 \leq \theta \leq \pi, 0 \leq \varphi<2 \pi\}$. We set $H=L_{2}(B)$, the space of real functions square integrable over $B$ endowed with the norm

$$
\|f\|^{2}=\int_{0}^{1} \int_{0}^{2 \pi} \int_{0}^{\pi}|f(r, \theta, \varphi)|^{2} r^{2} \sin \theta d \theta d \varphi d r .
$$

Our main tool will be expansion in eigenfunctions of the Laplace operator in $B$. For a function $f(r, \theta, \varphi) \in L_{2}(B)$ we can write

$$
f(r, \theta, \varphi)=\sum_{m \geq 0, n \geq 1} \widehat{f}_{m n} \chi_{m n}(r, \theta, \varphi),
$$

where $\chi_{m n}(r, \theta, \varphi)$ are the nontrivial solutions of the problem

$$
\begin{gathered}
\Delta \chi=-\Lambda \chi, \quad(r, \theta, \varphi) \in B, \\
\left.\chi\right|_{S}=0, \quad|\chi(0, \theta, \varphi)|<\infty, \quad \chi(r, \theta, \varphi+2 \pi)=\chi(r, \theta, \varphi),
\end{gathered}
$$

where $S=\partial B$ and

$$
\begin{aligned}
\Delta & =\left(1 / r^{2}\right) \partial_{r}\left(r^{2} \partial_{r}\right)+\left(1 / r^{2}\right) \Delta_{\theta, \varphi} \\
\Delta_{\theta, \varphi} & =(1 / \sin \theta) \partial_{\theta}\left(\sin \theta \partial_{\theta}\right)+\left(1 / \sin ^{2} \theta\right) \partial_{\varphi}^{2} .
\end{aligned}
$$


The angular eigenfunctions $Y(\theta, \varphi)$ come from the problem

$$
\begin{gathered}
\Delta_{\theta, \varphi} Y+\mu Y=0, \quad(\theta, \varphi) \in S, \\
|Y|_{\theta=0, \pi}<\infty, \quad Y(\theta, \varphi+2 \pi)=Y(\theta, \varphi),
\end{gathered}
$$

whose eigenvalues are $\mu_{m}=m(m+1), m=0,1,2, \ldots$ The functions $Y_{m}(\theta, \varphi)$ are called spherical harmonics of the $m$ th order, and they can be represented by the linear combination of tesseral harmonics [22]

$$
Y_{m}(\theta, \varphi)=\sum_{l=0}^{m}\left[C_{l m}^{(1)} \cos l \varphi+C_{l m}^{(2)} \sin l \varphi\right] P_{m}^{l}(\cos \theta),
$$

where $P_{m}^{l}(\cos \theta)$ are the associated Legendre functions.

The radial eigenfunctions are the nontrivial solutions of the problem

$$
\begin{gathered}
\frac{1}{r^{2}} \frac{d}{d r}\left(r^{2} \frac{d R}{d r}\right)+\left(\Lambda-\frac{m(m+1)}{r^{2}}\right) R=0, \\
|R(0)|<\infty, \quad R(1)=0 .
\end{gathered}
$$

They are called spherical Bessel functions and are defined by the formula

$$
R_{m n}(r)=j_{m}\left(\lambda_{m n} r\right)=\sqrt{\frac{\pi}{2 r}} J_{m+1 / 2}\left(\lambda_{m n} r\right) .
$$

Here $\Lambda_{m n}=\lambda_{m n}^{2}$ are the corresponding eigenvalues, $\lambda_{m n}$ are the positive zeros of the Bessel function $J_{m+1 / 2}(z)$ numbered in increasing order, $m=0,1,2, \ldots ; n=1,2, \ldots ; n$ is the number of the zero. We observe that $\lambda_{0 n}=\pi n$.

The Bessel functions $J_{\nu}\left(\lambda_{\nu n} r\right)$ are orthogonal and complete in the space $L_{2, r}(0,1)\left(L_{2}(0,1)\right.$ with the weight $\left.r\right)$ and for sufficiently large $\lambda>0[28$, p. 219],

$$
\frac{c_{1}}{\lambda} \leq \int_{0}^{1} r J_{\nu}^{2}(\lambda r) d r \leq \frac{c_{2}}{\lambda} .
$$

We introduce the real space $L_{2, r^{2}}(0,1)\left(L_{2}(0,1)\right.$ with the weight $\left.r^{2}\right)$ with the scalar product $(f, g)=\int_{0}^{1} r^{2} f(r) g(r) d r$ and norm $\|f\|^{2}=\int_{0}^{1} r^{2} f^{2}(r) d r$. Then we can write

$$
\left\|j_{m}\right\|_{(n)}^{2}=\int_{0}^{1} j_{m}^{2}\left(\lambda_{m n} r\right) r^{2} d r=\frac{\pi}{2} \int_{0}^{1} J_{m+1 / 2}^{2}\left(\lambda_{m n} r\right) r d r .
$$

The fact that we have used the same notation for the norm in $H$ and in $L_{2, r^{2}}(0,1)$ will not cause any confusion since in what follows, the latter will always be used with the subindex $n$ showing the dependence on $\lambda_{m n}$. 
Note that for sufficiently large $\lambda>0$,

$$
\frac{C_{1}}{\lambda} \leq \int_{0}^{1} j_{m}^{2}(\lambda r) r^{2} d r \leq \frac{C_{2}}{\lambda} .
$$

Large positive zeros of $J_{m}(z)$ with $0 \leq m \leq m_{0}<\infty$ have the following asymptotics uniform in $m$ (called McMahon's expansion, see [10]):

$$
\lambda_{m n}=\varrho_{m n}+O\left(\frac{1}{\varrho_{m n}}\right), \quad \varrho_{m n}=(m+2 n-1 / 2) \pi / 2, \quad n \rightarrow \infty .
$$

Next, we return to the angular eigenfunctions $Y_{m}(\theta, \varphi)$ and describe how they can be expressed in terms of zonal harmonics. This approach will be more advantageous than the use of (2.3) since it will simplify the calculation of $|\nabla u|^{2}$. Let $P$ and $Q$ be two variable points on the unit sphere $S$ and let $\gamma(P, Q)$ be the angle (between 0 and $\pi$ ) formed by two vector radii $O P$ and $O Q$, where $O$ is the center of the unit sphere. Then for $P$ fixed and $Q$ varying over $S, P_{m}[\cos \gamma(P, Q)]$ (where $P_{m}(x)$ is a Legendre polynomial) is a spherical harmonic of the $m$ th order of the spherical coordinates of $Q$, and for fixed $Q$ and variable $P$ this function is also a spherical harmonic with respect to $P$.

Introducing the scalar product in the real space $L_{2}(S)$ by the formula $(f, g)_{S}=\int_{S} f g d S$ and denoting by $\|\cdot\|_{S}$ the corresponding norm we have (see [22, p. 266])

$$
\begin{aligned}
& \left(Y_{m}, Y_{k}\right)_{S}=\int_{S} Y_{m}(Q) P_{k}[\cos \gamma(P, Q)] d S_{Q}=0, \quad m \neq k \\
& \left\|Y_{m}\right\|_{S}^{2}=\frac{4 \pi}{2 m+1}, \quad \frac{2 m+1}{4 \pi} \int_{S} Y_{m}(Q) P_{m}[\cos \gamma(P, Q)] d S_{Q}=Y_{m}(P) .
\end{aligned}
$$

The spherical harmonic expressed as a symmetric function of the two points $P$ and $Q$ is called a Laplace coefficient [22, p. 272], the name coming from the expansion of $f(P)$ into a Laplace series. Considered as a function of $Q$, $P_{m}[\cos \gamma(P, Q)]$ contains two arbitrary parameters, the coordinates $\left(\theta^{\prime}, \varphi^{\prime}\right)$ of the point $P$, which can be chosen by the choice of the coordinate system. If we direct the $z$-axis of the coordinate system through $P$, the spherical harmonics will become zonal and the constants will be determined. Then the last formula in (2.6) will yield

$$
\frac{2 m+1}{4 \pi} \int_{0}^{2 \pi} \int_{0}^{\pi}\left[P_{m}(\cos \gamma)\right]^{2} \sin \gamma d \gamma d \chi=Y_{m}(P)=1,
$$

where $(\gamma, \chi)$ are the spherical coordinates in the system with north pole 
at $P$. Another consequence of (2.6) is the formula

$$
\frac{2 m+1}{4 \pi} \int_{S} P_{m}\left[\cos \gamma\left(P, Q^{\prime}\right)\right] P_{m}\left[\cos \gamma\left(Q, Q^{\prime}\right)\right] d S_{Q^{\prime}}=P_{m}[\cos \gamma(P, Q)] .
$$

If $P=\left(\theta^{\prime}, \varphi^{\prime}\right)$ and $Q=(\theta, \varphi)$, then by the addition theorem for spherical harmonics we can represent them in terms of the tesseral ones

$$
\begin{aligned}
P_{m}[\cos \gamma(P, Q)]= & P_{m}\left[\cos \theta \cos \theta^{\prime}+\sin \theta \sin \theta^{\prime} \cos \left(\varphi-\varphi^{\prime}\right)\right] \\
= & P_{m}(\cos \theta) P_{m}\left(\cos \theta^{\prime}\right) \\
& +2 \sum_{l=1}^{m} \frac{(m-l) !}{(m+l) !} P_{m}^{l}(\cos \theta) P_{m}^{l}\left(\cos \theta^{\prime}\right) \cos \left[l\left(\varphi-\varphi^{\prime}\right)\right] .
\end{aligned}
$$

If we combine the north pole of the coordinate system with the point $P$, then $\theta^{\prime}=0, Y_{m}(P)=P_{m}(1)=1$, and $P_{m}[\cos \gamma(P, Q)]=P_{m}(\cos \theta)$.

As regards the eigenfunction expansion coefficients in $(2.1)$, we have shown in [36] that in the chosen coordinate system with north pole at $P$,

$$
\widehat{f}_{m n}=\frac{\left(\left(f, j_{m}\right)_{(n)}, Y_{m}\right)_{S}}{\left\|j_{m}\right\|_{(n)}^{2}\left\|Y_{m}\right\|_{S}^{2}}=\frac{\left\langle f, \chi_{m n}\right\rangle}{\left\|\chi_{m n}\right\|^{2}} .
$$

Now we shall give some facts concerning the Legendre polynomials $P_{m}(x),-1<x<1$ (see [22, pp. 176-200]). They satisfy the equation

$$
\frac{d}{d x}\left[(1-x)^{2} \frac{d}{d x} P_{m}(x)\right]+m(m+1) P_{m}(x)=0, \quad x \in(-1,1),
$$

and

$$
\left|P_{m}(x)\right|<1, \quad x \in(-1,1), \quad P_{m}(1)=1, \quad P_{m}(-1)=(-1)^{m} .
$$

First Theorem of Stieltues. For $\theta \in(0, \pi), m=1,2, \ldots$,

$$
\left|P_{m}(\cos \theta)\right| \leq \frac{4 \sqrt{2}}{\sqrt{\pi}} \cdot \frac{1}{\sqrt{m} \sqrt{\sin \theta}} .
$$

Second Theorem of Stieltues. For $x \in[-1,1], m=0,1, \ldots$,

$$
\left|P_{m+2}(x)-P_{m}(x)\right| \leq \frac{4}{\sqrt{\pi}} \cdot \frac{1}{\sqrt{m+2}} .
$$

We also have the relation

$$
\int_{-1}^{x} P_{m}(\xi) d \xi=\frac{P_{m+1}(x)-P_{m-1}(x)}{2 m+1}, \quad m \geq 1
$$

which implies that

$$
\left|\int_{-1}^{x} P_{m}(\xi) d \xi\right| \leq \frac{4}{\sqrt{\pi}} \cdot \frac{1}{\sqrt{m+1}(2 m+1)} .
$$


We introduce the Sobolev space $H^{s}(B)$ endowed with the equivalent norm

$$
\|f\|_{s}^{2}=\sum_{m \geq 0, n \geq 1} \lambda_{m n}^{2 s}\left|\widehat{f}_{m n}\right|^{2}\left\|\chi_{m n}\right\|^{2}
$$

and set $H_{0}^{s}(B)=H^{s}(B) \cap\left\{\left.u\right|_{S}=0\right\}$. We shall also use the Banach space $C^{0}\left([0, \infty), H_{0}^{s}(B)\right)$.

\section{Kuramoto-Sivashinsky equation in a ball. Statement of the} problem and the main results. We examine the first initial-boundary value problem for the Kuramoto-Sivashinsky equation in the unit ball $B$ :

$$
\begin{aligned}
& u_{t}+\nu \Delta^{2} u+\Delta u=|\nabla u|^{2}, \quad(r, \theta, \varphi) \in B, t>0, \\
& u(r, \theta, \varphi, 0)=\varepsilon^{2} \phi(r, \theta, \varphi), \quad(r, \theta, \varphi) \in B, \\
& |u(0, \theta, \varphi)|<\infty,\left.\quad u\right|_{S}=\left.\Delta u\right|_{S}=0, \\
& \text { periodicity conditions in } \varphi \text { with period } 2 \pi,
\end{aligned}
$$

where $\nu, \varepsilon=$ const $>0$ and $\phi(r, \theta, \varphi)$ is a real-valued function.

We set $A_{0}=-\Delta$ defined on sufficiently smooth functions satisfying the conditions (2.2). We also set $A=\nu A_{0}^{2}-A_{0}$ and note that $A>0$ for $\nu>1 / \lambda_{01}^{2}$, where $\lambda_{01}^{2}$ is the first eigenvalue of $A_{0}$.

Definition. The function $u(t)$ is called a mild solution of the problem (3.1) if it satisfies the integral equation (1.3) with $B(u(t), u(t))=|\sqrt{A} u(t)|^{2}$ in the Banach space $C^{0}\left([0, \infty), H_{0}^{s}(B)\right)$.

In what follows we shall use the notation $D_{\theta}=-(1 / \sin \theta) \partial_{\theta}$ and denote by $V_{0}^{1}(f(r, \theta, \varphi))$ the total variation of the function $f(r, \theta, \varphi)$ in $r \in[0,1]$. Now we formulate some assumptions on the function $f(r, Q), r \in(0,1)$, $Q=(\theta, \varphi) \in S$.

\section{Assumptions A.}

$$
\begin{gathered}
f(0, Q)=f(1, Q)=\partial_{r} f(0, Q)=\partial_{r} f(1, Q)=0 ; \\
D_{\theta}^{2} f(0, Q)=D_{\theta}^{2} f(1, Q)=\partial_{r} D_{\theta}^{2} f(0, Q)=\partial_{r} D_{\theta}^{2} f(1, Q)=0 ; \\
V_{0}^{1}\left(r \partial_{r}^{2} D_{\theta}^{2} f(0, Q)\right)=V_{2,2}(Q) \in L_{1}(S) \\
\lim _{r \rightarrow 0^{+}} r \partial_{r}^{2} D_{\theta}^{2} f(0, Q)=F_{2,2}(Q) \in L_{1}(S) .
\end{gathered}
$$

Our first result concerns the existence and uniqueness of global-in-time mild solutions of (3.1) and their representation in the form of eigenfunction expansion series.

Theorem 1. If $\nu>1 / \pi^{2}$ and the function $\phi(r, \theta, \varphi)$ satisfies Assumptions $\mathrm{A}$, then there is $\varepsilon_{0}>0$ such that for $\varepsilon \in\left[0, \varepsilon_{0}\right]$ there exists a mild solution of (3.1) in the space $C^{0}\left([0, \infty), H_{0}^{s}(B)\right)$ with $s<2$. It can be represented as 


$$
u(r, \theta, \varphi, t)=\sum_{m \geq 0, n \geq 1} \widehat{u}_{m n}(t) j_{m}\left(\lambda_{m n} r\right) Y_{m}(\theta, \varphi),
$$

where the coeffcients $\widehat{u}_{m n}(t)$ are defined by (1.8), (4.11). If $-1+\varepsilon \leq s<2$, where $\varepsilon>0$ is small, this solution is unique. If $3 / 2<s<2$, then $u \in$ $C_{\mathrm{b}}^{0}\left([0, \infty), C^{0}(B)\right)$.

REMARK 3.1. It is not difficult to give an example of an initial function satisfying Assumptions A. Using separation of variables we set $\phi(r, \theta, \varphi)=$ $R_{1}(r) \Theta_{1}(\theta) \Phi_{1}(\varphi)$ where $R_{1}, \Theta_{1}$ and $\Phi_{1}$ are defined on $(0,1),(0, \pi)$ and $(0,2 \pi)$ respectively and satisfy the following conditions:

$$
\begin{gathered}
R_{1}(0)=R_{1}(1)=R_{1}^{\prime}(0)=R_{1}^{\prime}(1)=0 ; \\
\lim _{r \rightarrow 0^{+}} r R_{1}^{\prime \prime}(r)=c_{3}<\infty, \quad V_{0}^{1}\left(r R_{1}^{\prime \prime}(r)\right)=c_{4}<\infty ; \\
\Phi_{1}(\varphi) \in L_{1}(0,2 \pi) \quad \text { and } \quad \frac{d}{d \theta}\left(\frac{1}{\sin \theta} \frac{d}{d \theta}\right) \Theta_{1}(\theta) \in L_{1}(0, \pi) .
\end{gathered}
$$

The next theorem is dedicated to obtaining the higher-order long-time asymptotics of the solution in question.

THEOREM 2. Under the assumptions of Theorem 1, there exists a constant $C$ such that for $s<2$ the following spatially uniform asymptotics holds:

$$
\left\|u(t)-\widetilde{u}_{0}(t)-\widetilde{u}_{1}(t)\right\|_{s} \leq C \exp \left(-3 \kappa_{01} t\right)
$$

where

$$
\begin{aligned}
\widetilde{u}_{0}(r, t)= & B_{01} \exp \left(-\kappa_{01} t\right) \frac{\sin (\pi r)}{\pi r}, \quad B_{01}=\sqrt{\pi} \sum_{N=0}^{\infty} \varepsilon^{N+1} A_{01}^{(N)}, \\
\widetilde{u}_{1}(r, \theta, \varphi, t)= & \exp \left(-2 \kappa_{01} t\right)\left[\widetilde{B}_{01} \frac{\sin (\pi r)}{\pi r}\right. \\
& \left.+\widetilde{A}\left(\sum_{m=0, n \geq 2}+\sum_{m, n \geq 1}\right) \frac{b(m, n, 0,1,0,1)}{\kappa_{m n}-2 \kappa_{01}} j_{m}\left(\lambda_{m n} r\right) Y_{m}(\theta, \varphi)\right],
\end{aligned}
$$

$\kappa_{01}=\pi^{2}\left(\nu \pi^{2}-1\right)>0, \kappa_{m n}=\lambda_{m n}^{2}\left(\nu \lambda_{m n}^{2}-1\right)>3 \kappa_{01}$ for $m=0, n \geq 2$ and for $m, n \geq 1$,

$$
\widetilde{B}_{01}=\sqrt{\pi} \frac{b(0,1,0,1,0,1)}{\kappa_{01}} \widetilde{A}, \quad \widetilde{A}=\sum_{N=1}^{\infty} \varepsilon^{N+1} \sum_{j=1}^{N} A_{01}^{(j-1)} A_{01}^{(N-j)},
$$

the coefficients $A_{01}^{(j)}$ are defined by (6.3), and the coefficients $b(m, n, 0,1,0,1)$ by (4.1).

4. Auxiliary results. In this section we collect several propositions that will permit us to estimate the eigenfunction expansion coefficients of 
the solution and the nonlinearity. We begin with calculating the latter. We have

$$
|\nabla u|^{2}=\sum_{m \geq 0, n \geq 1}\left(|\nabla u|^{2}\right)_{m n}^{\wedge}(t) \chi_{m n}(r, \theta, \varphi)
$$

where

$$
\begin{aligned}
\left(|\nabla u|^{2}\right)_{m n}^{\wedge}(t) & =\sum_{p, k \geq 0 ; q, s \geq 1} b(m, n, p, q, k, s) \widehat{u}_{p q}(t) \widehat{u}_{k s}(t), \\
b(m, n, p, q, k, s) & =\frac{\widetilde{b}(m, n, p, q, k, s)}{\left\|\chi_{m n}\right\|^{2}}=\frac{\left\langle\nabla \chi_{p q} \cdot \nabla \chi_{k s}, \chi_{m n}\right\rangle}{\left\|\chi_{m n}\right\|^{2}} .
\end{aligned}
$$

Since in spherical coordinates $\nabla=\left\{\partial_{r},(1 / r) \partial_{\theta},(1 / r \sin \theta) \partial_{\varphi}\right\}$, in the chosen coordinate system with north pole at $P$ (see Section 2 ) the third component of $\nabla u$ equals zero, and we have

$$
\widetilde{b}(m, n, p, q, k, s)=\left(\frac{\pi}{2}\right)^{3 / 2} \lambda_{p q} \lambda_{k s}\left[\left(\frac{1}{4} I_{1}+I_{2}+I_{3}+I_{4}\right) E_{1}+I_{1} E_{2}\right],
$$

where

$$
\begin{aligned}
I_{1} & =\frac{1}{4} \int_{0}^{1} \frac{J_{p+1 / 2}\left(\lambda_{p q} r\right)}{\lambda_{p q} r} \cdot \frac{J_{k+1 / 2}\left(\lambda_{k s} r\right)}{\lambda_{k s} r} J_{m+1 / 2}\left(\lambda_{m n} r\right) \sqrt{r} d r \\
I_{2} & =-\frac{1}{2} \int_{0}^{1} J_{p+1 / 2}^{\prime}\left(\lambda_{p q} r\right) \frac{J_{k+1 / 2}\left(\lambda_{k s} r\right)}{\lambda_{k s} r} J_{m+1 / 2}\left(\lambda_{m n} r\right) \sqrt{r} d r \\
I_{3} & =-\frac{1}{2} \int_{0}^{1} \frac{J_{p+1 / 2}\left(\lambda_{p q} r\right)}{\lambda_{p q} r} J_{k+1 / 2}^{\prime}\left(\lambda_{k s} r\right) \sqrt{r} d r \\
I_{4} & =\int_{0}^{1} J_{p+1 / 2}^{\prime}\left(\lambda_{p q} r\right) J_{k+1 / 2}^{\prime}\left(\lambda_{k s} r\right) J_{m+1 / 2}\left(\lambda_{m n} r\right) \sqrt{r} d r \\
E_{1} & =\int_{S} Y_{p}(Q) Y_{q}(Q) Y_{m}(Q) d S_{Q}, \\
E_{2} & =\int_{S} \partial_{\theta} Y_{p}(Q) \partial_{\theta} Y_{q}(Q) Y_{m}(Q) d S_{Q} .
\end{aligned}
$$

Using the formulas [38]

$$
J_{\mu}^{\prime}(z)=\frac{1}{2}\left[J_{\mu-1}(z)-J_{\mu+1}(z)\right], \quad \frac{J_{\mu}(z)}{z}=\frac{1}{2 \mu}\left[J_{\mu-1}(z)-J_{\mu+1}(z)\right]
$$

we deduce that

$$
\begin{aligned}
I_{1}= & \frac{1}{(2 p+1)} \cdot \frac{1}{(2 k+1)} \int_{0}^{1}\left[J_{p-1 / 2}\left(\lambda_{p q} r\right)+J_{p+3 / 2}\left(\lambda_{p q} r\right)\right] \\
& \times\left[J_{k-1 / 2}\left(\lambda_{k s} r\right)+J_{k+3 / 2}\left(\lambda_{k s} r\right)\right] J_{m+1 / 2}\left(\lambda_{m n} r\right) \sqrt{r} d r
\end{aligned}
$$




$$
\begin{aligned}
I_{2}= & -\frac{1}{4} \cdot \frac{1}{(2 k+1)} \int_{0}^{1}\left[J_{p-1 / 2}\left(\lambda_{p q} r\right)-J_{p+3 / 2}\left(\lambda_{p q} r\right)\right] \\
& \times\left[J_{k-1 / 2}\left(\lambda_{k s} r\right)+J_{k+3 / 2}\left(\lambda_{k s} r\right)\right] J_{m+1 / 2}\left(\lambda_{m n} r\right) \sqrt{r} d r \\
I_{3}= & -\frac{1}{4} \cdot \frac{1}{(2 p+1)} \int_{0}^{1}\left[J_{p-1 / 2}\left(\lambda_{p q} r\right)+J_{p+3 / 2}\left(\lambda_{p q} r\right)\right] \\
& \times\left[J_{k-1 / 2}\left(\lambda_{k s} r\right)-J_{k+3 / 2}\left(\lambda_{k s} r\right)\right] J_{m+1 / 2}\left(\lambda_{m n} r\right) \sqrt{r} d r \\
I_{4}= & \frac{1}{4} \int_{0}^{1}\left[J_{p-1 / 2}\left(\lambda_{p q} r\right)-J_{p+3 / 2}\left(\lambda_{p q} r\right)\right] \\
& \times\left[J_{k-1 / 2}\left(\lambda_{k s} r\right)-J_{k+3 / 2}\left(\lambda_{k s} r\right)\right] J_{m+1 / 2}\left(\lambda_{m n} r\right) \sqrt{r} d r, \\
E_{1}= & 2 \pi \int_{0}^{\pi} P_{p}(\cos \theta) P_{k}(\cos \theta) P_{m}(\cos \theta) \sin \theta d \theta, \\
E_{2}= & 2 \pi \int_{0}^{\pi} P_{p}^{\prime}(\cos \theta) P_{k}^{\prime}(\cos \theta) P_{m}(\cos \theta) \sin ^{3} \theta d \theta .
\end{aligned}
$$

Consider the integral

$$
\Im_{m}(\lambda, Q)=\int_{0}^{1} r^{3 / 2} f(r, Q) J_{m+3 / 2}(\lambda r) d r, \quad \lambda>0, Q \in S .
$$

The following proposition will allow us to estimate the eigenfunction coefficients of the initial data.

LEMma 1. Let $f(r, Q)$ have the partial derivative $\partial_{r}^{2} f(r, Q), r \in(0,1)$, $Q \in S$, and $f(0, Q)=f(1, Q)=\partial_{r} f(0, Q)=\partial_{r} f(1, Q)=0($ in case $m=0$ the condition $f(0, Q)=0$ is not needed). Moreover, assume that for each $Q \in S$ the function $r \partial_{r} f(r, Q)$ has bounded variation in $r \in[0,1]$ which is absolutely integrable over $\Pi$, i.e.,

$$
V_{0}^{1}\left(r \partial_{r}^{2} f(r, Q)\right)=V_{2,0}(Q) \in L_{1}(S), \quad \lim _{r \rightarrow 0^{+}} r \partial_{r}^{2} f(r, Q)=F_{2,0}(Q) \in L_{1}(S) .
$$

Then there exists $C_{Q} \in L_{1}(S)$ independent of $m$ and $\lambda$ such that for $m \geq 0$,

$$
\left|\Im_{m}(\lambda, Q)\right| \leq \frac{C_{Q}(m+1)^{2}}{\lambda^{7 / 2}} .
$$

Proof. The proof is a slight modification of that of Lemma 2 of [36] and is based on two-fold integration by parts in $r$ in the integral defining $\Im_{m}(\lambda, Q)$.

Next, we turn our attention to the typical integral appearing in the expressions $I_{i}, i=1,2,3,4$, i.e., we study 


$$
H_{m p k}\left(\lambda_{m n}, \lambda_{1}, \lambda_{2}\right)=\int_{0}^{1} J_{m+1 / 2}\left(\lambda_{m n} r\right) J_{p+1 / 2}\left(\lambda_{1} r\right) J_{k+1 / 2}\left(\lambda_{2} r\right) \sqrt{r} d r
$$

and obtain its estimate as $\lambda_{1}, \lambda_{2} \rightarrow \infty$. To this end we need a few facts concerning the Fresnel integrals [10, p. 28]

$$
C(x)=\frac{1}{\sqrt{2 \pi}} \int_{0}^{x} \frac{\cos t}{\sqrt{t}} d t, \quad S(x)=\frac{1}{\sqrt{2 \pi}} \int_{0}^{x} \frac{\sin t}{\sqrt{t}} d t .
$$

They have the following properties:

$$
\begin{gathered}
C(0)=S(0)=0, \quad C(\infty)=S(\infty)=1 / 2, \\
C(x)=\frac{1}{2}+\frac{\sin x}{\sqrt{2 \pi x}}+O\left(\frac{1}{x^{3 / 2}}\right), \\
S(x)=\frac{1}{2}-\frac{\cos x}{\sqrt{2 \pi x}}+O\left(\frac{1}{x^{3 / 2}}\right) \quad \text { as } x \rightarrow \infty .
\end{gathered}
$$

Lemma 2. For any fixed $n \geq 1$, any $m, p, k \geq 0$, and positive $\lambda_{1}, \lambda_{2} \rightarrow \infty$ there exists a constant $C$ independent of $m, n, p, k, \lambda_{1}, \lambda_{2}$ such that

$$
\left|H_{m p k}\left(\lambda_{m n}, \lambda_{1}, \lambda_{2}\right)\right| \leq C \begin{cases}\lambda_{1}^{-3 / 2} \lambda_{2}^{-1 / 2}, & \lambda_{1}>\lambda_{2}, \\ \lambda_{1}^{-1 / 2} \lambda_{2}^{-3 / 2}, & \lambda_{1}<\lambda_{2}, \\ \lambda^{-1}, & \lambda_{1}=\lambda_{2}=\lambda .\end{cases}
$$

Proof. We shall consider the case $\lambda_{1}>\lambda_{2}$ since $\lambda_{1}<\lambda_{2}$ can be examined analogously. For any fixed $n$ the function $J_{m+1 / 2}\left(\lambda_{m n} r\right)$ has $n+1$ intervals of monotonicity on the interval $[0,1]$ (see [39]). We shall denote them by $\left[0, r_{1}\right],\left[r_{1}, r_{2}\right], \ldots,\left[r_{i}, r_{i+1}\right], \ldots,\left[r_{n}, r_{n+1}\right]$ and the corresponding integrals by $H_{m p k}^{(i)}$. Then $H_{m p k}=\sum_{i=1}^{n+1} H_{m p k}^{(i)}$.

Since $J_{m+1 / 2}\left(\lambda_{m n} r\right) \geq 0$ and is increasing for $r \in\left[0, r_{1}\right]$ we can apply Bonnet's mean value theorem (see [26, p. 328]) to the corresponding integral and obtain

$$
H_{m p k}^{(1)}=J_{m+1 / 2}\left(\lambda_{m n} r_{1}\right) \int_{\eta}^{r_{1}} J_{p+1 / 2}\left(\lambda_{1} r\right) J_{k+1 / 2}\left(\lambda_{2} r\right) \sqrt{r} d r
$$

where $\eta \in\left(0, r_{1}\right)$. Note that $J_{m+1 / 2}\left(\lambda_{m n} r_{1}\right)$ is absolutely continuous on $\left[0, r_{1}\right]$.

We observe that for $\mu \geq 0$ and integer $l \geq 0$,

$$
\begin{gathered}
\left|J_{\mu}(x)\right| \leq \frac{C}{\sqrt{x}}, \quad x>0 . \\
J_{l+1 / 2}(x)=\sqrt{\frac{2}{\pi x}} \sin (x-l \pi / 2)+O\left(\frac{1}{x^{3 / 2}}\right), \quad x \rightarrow \infty .
\end{gathered}
$$


Next, we study the integral $\int_{\eta}^{r_{1}}$ in (4.3) and show that it has the bound $C / \lambda_{1}^{3 / 2} \lambda_{2}^{1 / 2}$ as $\lambda_{1}, \lambda_{2} \rightarrow \infty, \lambda_{1}>\lambda_{2}$. By (4.5), we deduce that

$$
\int_{\eta}^{r_{1}} J_{p+1 / 2}\left(\lambda_{1} r\right) J_{k+1 / 2}\left(\lambda_{2} r\right) \sqrt{r} d r=\widetilde{H}+O\left(\left(\lambda_{1} \lambda_{2}\right)^{-3 / 2}\right),
$$

where

$$
\widetilde{H}=\frac{2}{\pi \sqrt{\lambda_{1} \lambda_{2}}} \int_{\eta}^{r_{1}} \frac{\sin \left(\lambda_{1} r-p \pi / 2\right) \sin \left(\lambda_{2} r-k \pi / 2\right)}{\sqrt{r}} d r .
$$

We can rewrite $\widetilde{H}$ as

$$
\begin{aligned}
\widetilde{H}= & \frac{2}{\pi \sqrt{\lambda_{1} \lambda_{2}}}\left\{\cos [(p-k) \pi / 2] \int_{\eta}^{r_{1}} \frac{\cos \left(\Lambda_{-} r\right)}{\sqrt{r}} d r\right. \\
& \left.+\sin [(p-k) \pi / 2] \int_{\eta}^{r_{1}} \frac{\sin \left(\Lambda_{-} r\right)}{\sqrt{r}} d r\right\} \\
& -\cos [(p+k) \pi / 2] \int_{\eta}^{r_{1}} \frac{\cos \left(\Lambda_{+} r\right)}{\sqrt{r}} d r \\
& -\sin [(p+k) \pi / 2] \int_{\eta}^{r_{1}} \frac{\sin \left(\Lambda_{+} r\right)}{\sqrt{r}} d r
\end{aligned}
$$

where $\Lambda_{-}=\lambda_{1}-\lambda_{2}=\lambda_{1}\left(1-\lambda_{2} / \lambda_{1}\right) \rightarrow \infty$ as $\lambda_{1} \rightarrow \infty, \lambda_{1}>\lambda_{2}$; and $\Lambda_{+}=\lambda_{1}+\lambda_{2}$. Making the change of variable $\zeta=\Lambda_{-} r$ we obtain

$$
\begin{aligned}
\left|\int_{\eta}^{r_{1}} \frac{\cos \left(\Lambda_{-} r\right)}{\sqrt{r}} d r\right| & =\frac{1}{\sqrt{\Lambda_{-}}}\left|\int_{\Lambda_{-} \eta}^{\Lambda_{-} r_{1}} \frac{\cos \zeta}{\sqrt{\zeta}} d \zeta\right|=\sqrt{\frac{2 \pi}{\Lambda_{-}}}\left|C\left(\Lambda_{-} r\right)-C\left(\Lambda_{-} \eta\right)\right| \\
& \leq c / \Lambda_{-} \leq c / \lambda_{1} \quad \text { as } \lambda_{1} \rightarrow \infty
\end{aligned}
$$

The other integrals in (4.6) can be studied analogously. Using the uniform boundedness of the Bessel functions and recalling (4.4) we deduce the upper estimate in (4.2).

In order to estimate $H_{m p k}^{(i)}, i \geq 2$, we assume without loss of generality that the function $J_{m+1 / 2}\left(\lambda_{m n} r\right)$ is decreasing on $\left[r_{i}, r_{i+1}\right]$. We also observe that it is absolutely continuous on this interval. Then by the second mean value theorem there exists $\eta_{i} \in\left(r_{i}, r_{i+1}\right)$ such that

$$
\begin{aligned}
H_{m p k}^{(i)}= & J_{m+1 / 2}\left(\lambda_{m n} r_{i}\right) \int_{r_{i}}^{\eta_{i}} J_{p+1 / 2}\left(\lambda_{1} r\right) J_{k+1 / 2}\left(\lambda_{2} r\right) \sqrt{r} d r \\
& +J_{m+1 / 2}\left(\lambda_{m n} r_{i+1}\right) \int_{\eta_{i}}^{r_{i+1}} J_{p+1 / 2}\left(\lambda_{1} r\right) J_{k+1 / 2}\left(\lambda_{2} r\right) \sqrt{r} d r
\end{aligned}
$$




$$
\begin{aligned}
= & \frac{2}{\pi} \frac{1}{\sqrt{\lambda_{1} \lambda_{2}}}\left\{J_{m+1 / 2}\left(\lambda_{m n} r_{i}\right) \int_{r_{i}}^{\eta_{i}} \frac{\sin \left(\lambda_{1} r-p \pi / 2\right) \sin \left(\lambda_{2} r-k \pi / 2\right)}{\sqrt{r}} d r\right. \\
& +J_{m+1 / 2}\left(\lambda_{m n} r_{i+1}\right) \\
& \left.\times \int_{\eta_{i}}^{r_{i+1}} \frac{\sin \left(\lambda_{1} r-p \pi / 2\right) \sin \left(\lambda_{2} r-k \pi / 2\right)}{\sqrt{r}} d r+O\left(\frac{1}{\lambda_{1} \lambda_{2}}\right)\right\} .
\end{aligned}
$$

Conducting the same arguments as before we deduce that

$$
\left|H_{m p k}^{(i)}\right| \leq \frac{C}{\lambda_{1}^{3 / 2} \lambda_{2}^{1 / 2}}, \quad i \geq 2
$$

From these estimates, (4.2) follows for $\lambda_{1}<\lambda_{2}$.

Let $\lambda_{1}=\lambda_{2}=\lambda$. Applying the first mean value theorem for integrals (see [26]) and (4.4) we deduce that there exists $\xi \in(0,1)$ such that

$$
\left|H_{m p k}\right| \leq\left|J_{m+1 / 2}\left(\lambda_{m n} \xi\right)\right| \cdot \int_{0}^{1}\left|J_{p+1 / 2}\left(\lambda_{1} r\right)\right| \cdot\left|J_{k+1 / 2}\left(\lambda_{2} r\right)\right| \sqrt{r} d r \leq C \lambda^{-1}
$$

REMARK 4.1. An estimate analogous to that of (4.2) can be easily obtained for the integral

$$
\int_{0}^{1} J_{m+1 / 2}\left(\lambda_{m n} r\right) J_{-1 / 2}\left(\lambda_{1} r\right) J_{-1 / 2}\left(\lambda_{2} r\right) \sqrt{r} d r
$$

Such integrals appear in the expressions $I_{i}, i=1,2,3,4$, for $p=q=0$. Indeed, one can use the formula [28, p. 208]

$$
J_{-1 / 2}(x)=\sqrt{\frac{2}{\pi x}} \cos x
$$

and conduct the arguments described above.

By means of the change of variable $x=\cos \theta$ we can rewrite the integrals $E_{1,2}$ (see the beginning of this section) as

$$
\begin{aligned}
& E_{1}=2 \pi \int_{-1}^{1} P_{p}(x) P_{k}(x) P_{m}(x) d x \\
& E_{2}=2 \pi \int_{-1}^{1} P_{p}^{\prime}(x) P_{k}^{\prime}(x) P_{m}(x)\left(1-x^{2}\right) d x .
\end{aligned}
$$

Lemma 3. There exists a constant $C$ independent of $p, k, m$ such that for all integers $p, k, m \geq 0$,

$$
\left|E_{1}\right| \leq \frac{C}{\sqrt{(m+1)(p+1)(k+1)}}
$$

Proof. See [36, Lemma 3]. 
Lemma 4. There exists a constant $C$ independent of $p, k, m$ such that for all integers $p, k, m \geq 0$,

$$
\left|E_{2}\right| \leq C \frac{(p+1)(k+1)}{\sqrt{m+1}} .
$$

Proof. Using the equation for Legendre polynomials we can express $P_{p}^{\prime}(x)$ as follows:

$$
\begin{aligned}
P_{p}^{\prime}(x) & =-p(p+1) \frac{\int_{-1}^{x} P_{p}(\xi) d \xi}{1-x^{2}} \\
& =-\frac{p(p+1)}{2}\left[G_{p}^{(1)}(x)-G_{p}^{(2)}(x)\right], \quad-1<x<1,
\end{aligned}
$$

where

$$
G_{p}^{(1)}(x)=\frac{\int_{-1}^{x} P_{p}(\xi) d \xi}{1+x}, \quad G_{p}^{(2)}(x)=\frac{\int_{x}^{1} P_{p}(\xi) d \xi}{1-x} .
$$

Lagrange's mean value theorem applied to the intervals $[-1, x]$ and $[x, 1]$ yields

$$
G_{p}^{(1)}(x)=P_{p}\left(\eta_{1}\right), \quad \eta_{1} \in(-1, x) ; \quad G_{p}^{(2)}(x)=P_{p}\left(\eta_{2}\right), \quad \eta_{2} \in(x, 1) .
$$

Therefore, $\left|G_{p}^{(1,2)}(x)\right| \leq 1$ for all $x \in[-1,1]$ and integer $p \geq 0$. Consequently,

$$
\begin{aligned}
E_{2}= & 2 \pi \frac{p(p+1) k(k+1)}{4} \\
& \times \int_{-1}^{1}\left[G_{p}^{(1)}(x)-G_{p}^{(2)}(x)\right]\left[G_{k}^{(1)}(x)-G_{k}^{(2)}(x)\right] P_{m}(x) d x .
\end{aligned}
$$

Next, we fix some $0<\delta \leq 1$ and represent this integral in the following way:

$$
\begin{aligned}
E_{2} & =\Upsilon_{1}+\Upsilon_{2}+\Upsilon_{3} \\
& =2 \pi\left(\int_{-1}^{-1+\delta}+\int_{-1+\delta}^{1-\delta}+\int_{1-\delta}^{1}\right) P_{p}^{\prime}(x) P_{k}^{\prime}(x) P_{m}(x)\left(1-x^{2}\right) d x .
\end{aligned}
$$

Using (4.8), (2.8), and the boundedness of $G_{p}^{(1,2)}(x)$ we get

$$
\left|\Upsilon_{1,3}\right| \leq C \frac{p(p+1) k(k+1) \delta}{\sqrt{m+1}} .
$$

By means of the estimate [22]

$$
\left|P_{p}^{\prime}(x)\right| \leq C \frac{\sqrt{p+1}}{1-x^{2}}, \quad|x|<1, p \geq 0,
$$

we deduce that

$$
\left|\Upsilon_{2}\right| \leq C \sqrt{\frac{(p+1)(k+1)}{m+1}} \int_{-1+\delta}^{1-\delta} \frac{d x}{\left(1-x^{2}\right)^{5 / 4}} \leq C \sqrt{\frac{(p+1)(k+1)}{m+1}} \delta^{-1 / 4} .
$$


Choosing $\delta=\delta(p, k)=(p+1)^{-2}(k+1)^{-2}$ we deduce that

$$
\left|\Upsilon_{1,3}\right| \leq \frac{C}{\sqrt{m+1}}, \quad\left|\Upsilon_{2}\right| \leq C \frac{(p+1)(k+1)}{\sqrt{m+1}},
$$

which implies (4.8).

Lemma 5. If $f(r, Q)$ satisfies Assumptions A, then there exists a constant $C$ independent of $m, n$ such that for all integers $m \geq 0, n \geq 1$,

$$
\left|\widehat{f}_{m n}\right| \leq C \frac{\sqrt{m+1}}{\lambda_{m n}^{5 / 2}}
$$

Proof. First, we examine $m=0,1$. By Lemma 1, we have

$$
\begin{aligned}
\left|\widehat{f}_{m n}\right| & \leq c \lambda_{m n} \int_{0}^{2 \pi} d \varphi \int_{0}^{\pi}\left|P_{m}(\cos \theta)\right| \sin \theta d \theta\left|\int_{0}^{1} r^{2} j_{m}\left(\lambda_{m n} r\right) f(r, \theta, \varphi) d r\right| \\
& \leq C / \lambda_{m n}^{5 / 2} .
\end{aligned}
$$

Next we consider $m \geq 2$. Setting $z=\cos \theta$ we introduce the function

$$
\wp_{m}^{(2)}(z)=\int_{-1}^{z} d \xi \int_{-1}^{\xi} P_{m}(\eta) d \eta=\int_{-1}^{z}(z-\xi) P_{m}(\xi) d \xi
$$

and note that $\wp_{m}^{(2)}(1)=0$. Then, by $(2.10)$,

$$
\begin{aligned}
\wp_{m}^{(2)}(z) & =\frac{1}{2 m+1}\left[\int_{-1}^{z} P_{m+1}(\xi) d \xi-\int_{-1}^{z} P_{m-1}(\xi) d \xi\right] \\
& =\frac{1}{2 m+1}\left[\frac{P_{m+2}(z)-P_{m}(z)}{2 m+3}-\frac{P_{m}(z)-P_{m-2}(z)}{2 m-1}\right], \quad m \geq 2 .
\end{aligned}
$$

Hence, by (2.9) and (2.11),

$$
\left|\wp_{m}^{(2)}(z)\right| \leq C / m^{5 / 2}, \quad m \geq 2 .
$$

Now we study the integral

$$
\Gamma_{m}(r)=\int_{0}^{\pi} F(r, \cos \theta) P_{m}(\cos \theta) \sin \theta d \theta=\int_{-1}^{1} F(r, z) P_{m}(z) d z,
$$

where

$$
F(r, z)=F(r, \cos \theta)=\langle f\rangle(r, \theta)=\frac{1}{2 \pi} \int_{0}^{2 \pi} f(r, \theta, \varphi) d \varphi
$$

is the mean value of the function $f(r, \theta, \varphi)$ along the parallel all of whose points have colatitude $\theta$. Each plane characterized by the condition $\theta=$ const has a distance $z=\cos \theta$ from the center of the unit sphere $S$. 
Integrating two times by parts we get

$$
\Gamma_{m}(r)=\int_{-1}^{1} \wp_{m}^{(2)}(z) \partial_{z}^{2} F(r, z) d z=\int_{0}^{\pi} \wp_{m}^{(2)}(\cos \theta) D_{\theta}^{2} F(r, \cos \theta) \sin \theta d \theta
$$

Since $D_{\theta}^{2} f(r, \theta, \varphi)$ satisfies the hypotheses of Lemma 1 , we obtain

$$
\begin{aligned}
\left|\widehat{f}_{m n}\right| & \leq C \lambda_{m n}(2 m+1) \int_{0}^{2 \pi} d \varphi \int_{0}^{\pi}\left|\wp_{m}^{(2)}(\cos \theta)\right| d \theta \\
& \leq\left|\int_{0}^{1} r^{2} j_{m}\left(\lambda_{m n} r\right) \partial_{\theta}\left(\frac{1}{\sin \theta} \partial_{\theta}\right) f(r, \theta, \varphi) d r\right| \\
& \leq \frac{C(m+1)^{2}(2 m+1) \lambda_{m n}}{\lambda_{m n}^{7 / 2} m^{5 / 2}} \leq C \frac{\sqrt{m+1}}{\lambda_{m n}^{5 / 2}}
\end{aligned}
$$

The next proposition serves to estimate the linear and nonlinear iterations of the type of (1.9) which for the problem in question can be written as

$$
\begin{aligned}
\widehat{v}_{m n}^{(0)}(t)= & \widehat{\Phi}_{m n} \exp \left(-\kappa_{m n} t\right) \\
\widehat{v}_{m n}^{(N)}(t)= & \int_{0}^{t} \exp \left[-\kappa_{m n}(t-\tau)\right] \\
& \times \sum_{p, k \geq 0 ; q, s \geq 1} b(m, n, p, q, k, s) \sum_{j=1}^{N} \widehat{v}_{p q}^{(j-1)}(\tau) \widehat{v}_{k s}^{(N-j)}(\tau) d \tau
\end{aligned}
$$

where $\widehat{\Phi}_{m n}=\varepsilon \widehat{\phi}_{m n}$ and the coefficients $b(m, n, p, q, k, s)$ are defined by (4.1).

LEMMA 6. For the functions $\widehat{v}_{m n}^{(N)}(t)$ the following estimates hold for integers $m \geq 0, n \geq 1, N \geq 0$, and real $t \geq 0$ :

$$
\left|\widehat{v}_{m n}^{(N)}(t)\right| \leq c^{N}(N+1)^{-2} \lambda_{m n}^{-5 / 2} \sqrt{m+1} \exp \left(-\kappa_{01} t\right) .
$$

Proof. Since the function $\phi(r, \theta, \varphi)$ satisfies Assumptions A, its eigenfunction expansion coefficients $\widehat{\phi}_{m n}$ satisfy (4.10). We use induction on $N$. For $N=0$ and sufficiently small $\varepsilon$ we have

$$
\left|\widehat{v}_{m n}^{(N)}(t)\right| \leq \varepsilon\left|\widehat{\phi}_{m n}\right| \exp \left(-\kappa_{m n} t\right) \leq \lambda_{m n}^{-5 / 2} \sqrt{m+1} \exp \left(-\kappa_{01} t\right) .
$$

We assume that (4.12) is valid for all $\widehat{v}_{m n}^{(s)}(t)$ with $0 \leq s \leq N-1$ and prove that it holds for $s=N$. For this purpose we need the inequality (see $[17$, p. 181])

$$
j^{-2}(N+1-j)^{-2} \leq 2^{2}(N+1)^{-2}\left[j^{-2}+(N+1-j)^{-2}\right], \quad 1 \leq j \leq N .
$$


According to (4.11), we have

$$
\begin{aligned}
\left|\widehat{v}_{m n}^{(N)}(t)\right| \leq & \int_{0}^{t} \exp \left[-\kappa_{m n}(t-\tau)\right] \\
& \times \sum_{p, k \geq 0 ; q, s \geq 1}|b(m, n, p, q, k, s)|\left|\sum_{j=1}^{N} \widehat{v}_{p q}^{(j-1}(\tau) \widehat{v}_{k s}^{(N-j)}(\tau)\right| d \tau \\
\leq & c L_{m n}(t) S_{N} \\
& \times \sum_{p, k \geq 0 ; q, s \geq 1}|b(m, n, p, q, k, s)| \lambda_{p q}^{-5 / 2} \lambda_{k s}^{-5 / 2} \sqrt{p+1} \sqrt{k+1}
\end{aligned}
$$

where

$$
\begin{aligned}
L_{m n}(t) & =\exp \left(-\kappa_{m n} t\right) \int_{0}^{t} \exp \left[\left(\kappa_{m n}-2 \kappa_{01}\right) \tau\right] d \tau \\
S_{N} & =\sum_{j=1}^{N} c^{j-1} c^{N-j} j^{-2}(N+1-j)^{-2} \leq c^{N-1}(N+1)^{-2} .
\end{aligned}
$$

By Lemmas 2-4 and (4.1), (2.4), (2.6), we deduce that for $n \geq 1$; $m, p, k \geq 0 ; q \geq q_{0}>0, s \geq s_{0}>0\left(q_{0}, s_{0}\right.$ being sufficiently large $)$,

$$
|b(m, n, p, q, k, s)| \leq C \frac{\lambda_{p q} \lambda_{k s} \lambda_{m n}(2 m+1)}{\sqrt{(m+1)(p+1)(k+1)}} \begin{cases}\lambda_{p q}^{-3 / 2} \lambda_{k s}^{-1 / 2}, & \lambda_{p q}>\lambda_{k s} \\ \lambda_{p q}^{-1 / 2} \lambda_{k s}^{-3 / 2}, & \lambda_{p q}<\lambda_{k s} \\ \lambda_{p q}^{-1}, & \lambda_{p q}=\lambda_{k s} .\end{cases}
$$

Therefore,

$$
\begin{aligned}
\left|\widehat{v}_{m n}^{(N)}(t)\right| \leq & c L_{m n}(t) S_{N} \frac{\lambda_{m n}(2 m+1)}{\sqrt{m+1}}\left\{\sum_{\substack{p, q, k, s: \\
\lambda_{p q}>\lambda_{k s}}} \frac{\lambda_{p q} \lambda_{k s}}{\lambda_{p q}^{5 / 2} \lambda_{k s}^{5 / 2} \lambda_{p q}^{3 / 2} \lambda_{k s}^{1 / 2}}\right. \\
& \left.+\sum_{\substack{p, q, k, s: \\
\lambda_{p q}>\lambda_{k s}}} \frac{\lambda_{p q} \lambda_{k s}}{\lambda_{p q}^{5 / 2} \lambda_{k s}^{5 / 2} \lambda_{p q}^{1 / 2} \lambda_{k s}^{3 / 2}}+\sum_{p, q} \frac{1}{\lambda_{p q}^{4}}\right\} \\
\leq & c L_{m n}(t) S_{N} \frac{\lambda_{m n}(2 m+1)}{\sqrt{m+1}}\left(2 \sum_{p, q} \frac{1}{\lambda_{p q}^{5 / 2}} \sum_{k, s} \frac{1}{\lambda_{k s}^{5 / 2}}+\sum_{p, q} \frac{1}{\lambda_{p q}^{4}}\right) \\
\leq & c^{N}(N+1)^{-2} L_{m n}(t) \lambda_{m n} \sqrt{m+1} .
\end{aligned}
$$

Next, we prove that

$$
L_{m n}(t) \leq C \frac{\exp \left(-\kappa_{01} t\right)}{\lambda_{m n}^{4}} .
$$


(i) If $m=0, n=1$, then

$$
\begin{aligned}
L_{m n}(t) & =\exp \left(-\kappa_{01} t\right) \int_{0}^{t} \exp \left(-\kappa_{01} \tau\right) d \tau=\exp \left(-\kappa_{01} t\right) \frac{1-\exp \left(-\kappa_{01} t\right)}{\kappa_{01}} \\
& \leq \frac{\exp \left(-\kappa_{01} t\right)}{\lambda_{01}^{4}\left(\nu-1 / \lambda_{01}^{4}\right)} .
\end{aligned}
$$

(ii) If $m=0, n \geq 2$, then $\lambda_{0 n}=\pi n$ and $\lambda_{0 n}^{2}-2 \lambda_{01}^{2} \geq \lambda_{02}^{2}-2 \lambda_{01}^{2}=2 \pi^{2}$, therefore,

$$
\begin{aligned}
\kappa_{0 n}- & 2 \kappa_{01}=\nu \lambda_{0 n}^{2}\left(\lambda_{0 n}^{2}-\lambda_{01}^{2}\right)+\left(\nu \lambda_{01}^{2}-1\right)\left(\lambda_{0 n}^{2}-2 \lambda_{01}^{2}\right)>0, \\
L_{m n}(t) & =\exp \left(-\kappa_{0 n} t\right) \frac{\exp \left[\left(\kappa_{0 n}-2 \kappa_{01}\right) t\right]-1}{\kappa_{0 n}-2 \kappa_{01}} \leq \frac{\exp \left(-2 \kappa_{01} t\right)}{\nu \lambda_{0 n}^{4}\left(1-\lambda_{01}^{2} / \lambda_{0 n}^{2}\right)} \\
& \leq C(\nu) \frac{\exp \left(-\kappa_{01} t\right)}{\lambda_{0 n}^{4}} .
\end{aligned}
$$

(iii) If $m=0, n=1$, then $\lambda_{m n}^{2}-2 \lambda_{01}^{2} \geq \lambda_{11}^{2}-2 \lambda_{01}^{2}>0$ since $\lambda_{11} \simeq 4.493$ and $\lambda_{01}=\pi$ (see [10]). Consequently, $\kappa_{m n}-2 \kappa_{01}>0$ and

$$
\begin{aligned}
L_{m n}(t) & =\exp \left(-\kappa_{m n} t\right) \frac{\exp \left[\left(\kappa_{m n}-2 \kappa_{01}\right) t\right]-1}{\kappa_{m n}-2 \kappa_{01}} \\
& \leq \frac{\exp \left(-2 \kappa_{01} t\right)}{\nu \lambda_{m n}^{4}\left(1-\lambda_{01}^{2} / \lambda_{m n}^{2}\right)} \leq C(\nu) \frac{\exp \left(-\kappa_{01} t\right)}{\lambda_{0 n}^{4}} .
\end{aligned}
$$

Thus, (4.14) is established. Combining (4.13) and (4.14) we deduce the required estimate.

Corollary. For $t \geq 0, N \geq 1$ and for $m=0, n \geq 2$ and $m, n \geq 1$,

$$
\left|\widehat{v}_{m n}^{(N)}(t)\right| \leq c^{N}(N+1)^{-2} \lambda_{m n}^{-5 / 2} \sqrt{m+1} \exp \left(-2 \kappa_{01} t\right) .
$$

Proof. Since $\kappa_{0 n} \geq \kappa_{02}>2 \kappa_{01}$ for $n \geq 2$ and $\kappa_{m n} \geq \kappa_{11}>2 \kappa_{01}$ for $m, n \geq 1,(4.15)$ for $N=0$ is evident. Then we apply induction on $N$ and repeating the arguments of (ii) and (iii) above we arrive at (4.15).

\section{Proof of Theorem 1}

5.1. Existence and construction of solutions. We seek mild solutions of (3.1) in the form of an eigenfunction expansion series

$$
u(r, \theta, \varphi, t)=\sum_{m \geq 0, n \geq 1} \widehat{u}_{m n}(t) \chi_{m n}(r, \theta, \varphi),
$$

where

$$
\widehat{u}_{m n}(t)=\frac{\left\langle u, \chi_{m n}\right\rangle(t)}{\left\|\chi_{m n}\right\|^{2}}, \quad \chi_{m n}=j_{m}\left(\lambda_{m n} r\right) Y_{m}(\theta, \varphi) .
$$

Expanding the nonlinearity $|\nabla u|^{2}$ in a series of the type of (5.1) with $\left(|\nabla u|^{2}\right)_{m n}^{\wedge}(t)$ defined by (4.1) we substitute the result and (5.1) into (3.1) 
to get

$$
\begin{aligned}
& \widehat{u}_{m n}^{\prime}(t)+\kappa_{m n} \widehat{u}_{m n}(t)=\left(|\nabla u|^{2}\right)_{m n}^{\wedge}(t), \quad t>0 \\
& \widehat{u}_{m n}(0)=\varepsilon^{2} \widehat{\phi}_{m n}, \quad \kappa_{m n}=\lambda_{m n}^{2}\left(\nu \lambda_{m n}^{2}-1\right)>0
\end{aligned}
$$

where $\widehat{\phi}_{m n}$ are the coefficients of the eigenfunction expansion of the initial function, i.e.,

$$
\phi(r, \theta, \varphi)=\sum_{m \geq 0, n \geq 1} \widehat{\phi}_{m n} \chi_{m n}(r, \theta, \varphi), \quad \widehat{\phi}_{m n}=\frac{\left\langle\phi, \chi_{m n}\right\rangle}{\left\|\chi_{m n}\right\|^{2}} .
$$

Setting $\widehat{\Phi}_{m n}=\varepsilon \widehat{\phi}_{m n}$ (it is convenient to keep $\varepsilon$ in the coefficients in order to simplify some estimates) we integrate the Cauchy problem (5.2) in $t$ to obtain

$$
\widehat{u}_{m n}(t)=\varepsilon \widehat{\Phi}_{m n} \exp \left(-\kappa_{m n} t\right)+\int_{0}^{t} \exp \left[-\kappa_{m n}(t-\tau)\right]\left(|\nabla u|^{2}\right)_{m n}^{\wedge}(\tau) d \tau
$$

Represent $\widehat{u}_{m n}(t)$ as a formal series in $\varepsilon$ (see (1.8)) and substitute it into (5.3) to obtain the recurrence formulas (4.11) for $\widehat{v}_{m n}^{(N)}(t)$. By Lemma 6 , the inequalities (4.12) hold for $\widehat{v}_{m n}^{(N)}(t)$ with $m \geq 0, n \geq 1$ and the estimates (4.15) for these functions with $m=0, n \geq 2$ and with $m, n \geq 1$.

Next, we prove that the formally constructed function $(5.1),(1.8),(4.11)$ is really a mild solution of (3.1) from the space $C^{0}\left([0, \infty), H^{s}(B)\right), s<2$. Choosing $\varepsilon \in\left[0, \varepsilon_{0}\right], \varepsilon_{0}<1 / c$, where $c$ is the constant which appears in the estimates (4.12), we deduce by virtue of (1.8) that for $m \geq 0, n \geq 1$,

$$
\left|\widehat{u}_{m n}(t)\right| \leq c \lambda_{m n}^{-5 / 2} \sqrt{m+1} \exp \left(-\kappa_{01} t\right) .
$$

Using (2.4)-(2.6) and (5.4) we deduce that the series

$$
\|u(t)\|_{s}^{2}=\sum_{m \geq 0, n \geq 1} \lambda_{m n}^{2 s}\left|\widehat{u}_{m n}(t)\right|^{2}\left\|Y_{m}\right\|_{S}^{2}\left\|j_{m}\right\|_{(n)}^{2}
$$

converges absolutely and uniformly with respect to $t \geq 0$ for $s<2$. To this end we apply the Fubini-Tonelli theorem to establish the convergence of the iterated series $\sum_{m} \sum_{n}$ by comparison with the integral

$$
\int_{C}^{\infty} \frac{(m+1) d m}{2 m+1} \int_{D}^{\infty} \frac{d n}{(m+2 n)^{6-2 s}}
$$

with sufficiently large $C, D>0$. By the Sobolev embedding theorem and $(5.4), u(t):[0, \infty) \rightarrow C^{0}(B)$ is continuous and bounded for $3 / 2<s<2$.

5.2. Uniqueness of solutions. We shall argue by contradiction. Assume that there exist two mild solutions $u^{(1)}$ and $u^{(2)}$ of problem (3.1) from the class stated in the theorem. Then each can be expanded in a series (5.1), where the coefficients $\widehat{u}_{m n}^{(i)}(t), i=1,2$, satisfy (5.4). Set $w=u^{(1)}-u^{(2)}$ and 
expand it in a series of the type (5.1):

$$
\begin{aligned}
w(r, \theta, \varphi, t)= & \sum_{m \geq 0, n \geq 1} \widehat{w}_{m n}(t) j_{m}\left(\lambda_{m n} r\right) Y_{m}(\theta, \varphi), \\
\widehat{w}_{m n}(t)= & \int_{0}^{t} \exp \left[-\kappa_{m n}(t-\tau)\right] \sum_{p, q, k, s} b(m, n, p, q, k, s) \\
& \times\left[\widehat{u}_{p q}^{(1)}(\tau) \widehat{w}_{k s}(\tau)+\widehat{u}_{k s}^{(2)}(\tau) \widehat{w}_{p q}(\tau)\right] d \tau .
\end{aligned}
$$

We can estimate the nonlinear terms as follows:

$$
\left|\sum_{p, q, k, s} b(m, n, p, q, k, s) \widehat{u}_{p q}^{(1)}(t) \widehat{w}_{k s}(t)\right| \leq C \frac{(2 m+1) \lambda_{m n}}{\sqrt{m+1}}\left[\Sigma_{1}+\Sigma_{2}+\Sigma_{3}\right],
$$

where

$$
\begin{aligned}
& \Sigma_{1}=\sum_{\substack{p, q, k, s: \\
\lambda_{p q}>\lambda_{k s}}} \frac{\left|\widehat{u}_{p q}^{(1)}(t)\right|}{\sqrt{(p+1)(k+1)}} \cdot \frac{\left|\widehat{w}_{k s}(t)\right|}{\lambda_{p q}^{3 / 2} \lambda_{k s}^{1 / 2}}, \\
& \Sigma_{2}=\sum_{\substack{p, q, k, s: \\
\lambda_{p q}<\lambda_{k s}}} \frac{\left|\widehat{u}_{p q}^{(1)}(t)\right|}{\sqrt{(p+1)(k+1)}} \cdot \frac{\left|\widehat{w}_{k s}(t)\right|}{\lambda_{p q}^{1 / 2} \lambda_{k s}^{3 / 2}}, \\
& \Sigma_{3}=\sum_{p, q} \frac{\left|\widehat{u}_{p q}^{(1)}(t)\right|}{p+1} \cdot \frac{\left|\widehat{w}_{p q}(t)\right|}{\lambda_{p q}} .
\end{aligned}
$$

We estimate only the sum $\Sigma_{1}$ since $\Sigma_{2,3}$ can be treated analogously. By virtue of the Cauchy-Schwarz inequality and (5.6), for some small $\varepsilon_{1}>0$,

$$
\begin{aligned}
\left|\Sigma_{1}\right| & =\left|\sum_{k, s} \sqrt{\frac{2 k+1}{k+1}} \cdot \frac{1}{\lambda_{k s}^{2+\gamma-\varepsilon_{1}}} \cdot \frac{\lambda_{k s}^{\gamma}\left|\widehat{w}_{k s}(t)\right|}{\sqrt{2 k+1} \lambda_{k s}^{1 / 2}} \cdot \sum_{p, q} \frac{1}{\lambda_{p q}^{3 / 2+\varepsilon_{1}} \sqrt{p+1}}\right| \\
& \leq C\left(\sum_{k, s} \frac{1}{\lambda_{k s}^{2 \gamma+4-2 \varepsilon_{1}}}\right)^{1 / 2}\left(\sum_{k, s} \lambda_{k s}^{2 \gamma}\left\|Y_{k}\right\|_{S}^{2}\left\|j_{k}\right\|_{(s)}^{2}\left|\widehat{w}_{k s}(t)\right|^{2}\right)^{1 / 2} .
\end{aligned}
$$

Here the series over $p, q$ converges for any $\varepsilon_{1}>0$. The first series over $k, s$ on the right-hand side converges if $2 \gamma+4-2 \varepsilon_{1}>2$, i.e., if $\gamma \geq-1+\varepsilon_{1}$, where $\varepsilon_{1}>0$. The second series over $k, s$ represents $\|w(t)\|_{\gamma}$ and converges for $\gamma<2$. Thus, for $-1+\varepsilon_{1} \leq \gamma<2$ we obtain

$$
\left|\Sigma_{1}\right| \leq C\|w(t)\|_{\gamma} .
$$

Consequently,

$$
\left|\widehat{w}_{m n}(t)\right| \leq C \int_{0}^{t} \exp \left[-\kappa_{m n}(t-\tau)\right]\|w(\tau)\|_{\gamma} d \tau
$$


Squaring both sides of the last inequality, multiplying the result by $\lambda_{m n}^{2 \gamma}\left\|Y_{m}\right\|_{S}^{2}\left\|j_{m}\right\|_{(n)}^{2}$ and summing over $m, n$ we deduce that for some $h>0$ and $t \in[0, h]$,

$$
\left\|\widehat{w}_{m n}(t)\right\|_{\gamma}^{2} \leq C\left(\sup _{t \in[0, h]}\left\|\widehat{w}_{m n}(t)\right\|_{\gamma}^{2}\right) Q(t)
$$

where

$$
Q(t)=\sum_{m \geq 0, n \geq 1} \frac{\left[1-\exp \left(-\kappa_{m n} t\right)\right]^{2} \lambda_{m n}^{2 \gamma}}{\kappa_{m n}^{2} \lambda_{m n}(2 m+1)} .
$$

The series $Q(t)$ converges absolutely and uniformly with respect to $t \in[0, h]$ for $\gamma<4$. It is a continuous nondecreasing function on $[0, h]$ and $Q(0)=0$. Therefore, for $-1+\varepsilon_{1} \leq \gamma<2$,

$$
\left(\sup _{t \in[0, h]}\left\|\widehat{w}_{m n}(t)\right\|_{\gamma}\right)^{2} \leq C Q(t)\left(\sup _{t \in[0, h]}\left\|\widehat{w}_{m n}(t)\right\|_{\gamma}\right)^{2} \leq C(h)\left(\sup _{t \in[0, h]}\left\|\widehat{w}_{m n}(t)\right\|_{\gamma}\right)^{2},
$$

where $C(h)=C Q(h)$. The constant $C(h)$ can be made less than one by an appropriate choice of $h$. This contradiction establishes uniqueness for $t \in[0, h]$.

Next, we consider the sequence $\left\{\left[T_{k}, T_{k+1}\right]\right\}_{k=1}^{\infty}$ of intervals with $T_{k}=k h$. Since

$$
\int_{T_{k}}^{t} \exp \left[-\kappa_{m n}(t-\tau)\right] d \tau=\frac{1-\exp \left[-\kappa_{m n}\left(t-T_{k}\right)\right]}{\kappa_{m n}},
$$

arguing as above we obtain, for $t \in\left[T_{k}, T_{k+1}\right]$,

$$
\left(\sup _{t \in\left[T_{k}, T_{k+1}\right]}\left\|\widehat{w}_{m n}(t)\right\|_{\gamma}\right)^{2} \leq C Q\left(t-T_{k}\right)\left(\sup _{t \in\left[T_{k}, T_{k+1}\right]}\left\|\widehat{w}_{m n}(t)\right\|_{\gamma}\right)^{2} .
$$

Setting $t=T_{k}+\eta, \eta \in[0, h]$, we deduce that $Q\left(t-T_{k}\right)=Q(\eta)<1$. Thus, we have established the uniqueness for all $t \geq 0$ and $-1+\varepsilon_{1} \leq \gamma<2$.

6. Proof of Theorem 2: long-time asymptotics. We can represent the solution in question as

$$
\begin{aligned}
u(r, \theta, \varphi, t)= & \widehat{u}_{01}(t) j_{0}\left(\lambda_{01} r\right) \\
& +\left(\sum_{m=0, n \geq 2}+\sum_{m, n \geq 1}\right) \widehat{u}_{m n}(t) j_{m}\left(\lambda_{m n} r\right) Y_{m}(\theta, \varphi) .
\end{aligned}
$$

First, we obtain a subtle asymptotic estimate of the coefficient $\widehat{u}_{01}(t)$ which contributes to the major term of the long-time asymptotics, and then we single out the major parts as $t \rightarrow \infty$ of $\widehat{u}_{m n}(t)$ with $m \neq 0, n \neq 1$. We have

$$
\widehat{u}_{01}(t)=\sum_{N=0}^{\infty} \varepsilon^{N+1} \widehat{v}_{01}^{(N)}(t),
$$

where $\widehat{v}_{01}^{(N)}(t)$ can be represented as follows: 


$$
\begin{aligned}
\widehat{v}_{01}^{(0)}(t)= & A_{01}^{(0)} \exp \left(-\kappa_{01} t\right), \\
\widehat{v}_{01}^{(N)}(t)= & \exp \left(-\kappa_{01} t\right)\left[A_{01}^{(N)}+R_{01}^{(N)}(t)\right], \quad N \geq 1, \\
A_{01}^{(0)}= & \widehat{\Phi}_{01}=\varepsilon \widehat{\phi}_{01}, \\
A_{01}^{(N)}= & \int_{0}^{\infty} \exp \left(\kappa_{01} \tau\right) \\
& \times \sum_{p, k \geq 0 ; q, s \geq 1} b(0,1, p, q, k, s) \sum_{j=1}^{N} \widehat{v}_{p q}^{(j-1)}(\tau) \widehat{v}_{k s}^{(N-j)}(\tau) d \tau,
\end{aligned}
$$

$$
\begin{aligned}
R_{01}^{(N)}(t)= & -\int_{t}^{\infty} \exp \left(\kappa_{01} \tau\right) \\
& \times \sum_{p, k \geq 0 ; q, s \geq 1} b(0,1, p, q, k, s) \sum_{j=1}^{N} \widehat{v}_{p q}^{(j-1)}(\tau) \widehat{v}_{k s}^{(N-j)}(\tau) d \tau
\end{aligned}
$$

and the functions $\widehat{v}_{m n}^{(j)}(\tau), j=0,1, \ldots, N-1$, are defined by (4.11). Here we have added and subtracted the integrals from $t$ to $\infty$ in the integral representations for $\widehat{v}_{01}^{(N)}(t), N \geq 1$.

Next, we estimate the residual term $R_{01}^{(N)}(t)$ by means of (4.11), (4.15). We have

$$
\left|R_{01}^{(N)}(t)\right| \leq c S_{N} \int_{t}^{\infty} \exp \left(\kappa_{01} \tau\right)\left[C_{1} \exp \left(-2 \kappa_{01} \tau\right)+C_{2} \exp \left(-4 \kappa_{01} \tau\right)\right] d \tau,
$$

where $S_{N}$ was defined in the proof of Lemma 6 . Therefore,

$$
\left|R_{01}^{(N)}(t)\right| \leq c^{N}(N+1)^{-2} \exp \left(-\kappa_{01} t\right) .
$$

Now we obtain the second-order asymptotics of $\widehat{u}_{01}(t)$. Substituting (6.3), (6.5) into (6.4) and using (4.15) we get

$$
\begin{aligned}
R_{01}^{(N)}(t)= & -\int_{t}^{\infty} \exp \left(\kappa_{01} \tau\right)\left[\exp \left(-2 \kappa_{01} \tau\right) b(0,1,0,1,0,1) \sum_{j=1}^{N} A_{01}^{(j-1)} A_{01}^{(N-j)}\right. \\
& +c^{N}(N+1)^{-2} O\left(\exp \left(-3 \kappa_{01} \tau\right)\right) \\
& \left.+\sum_{p, k \geq 1 ; q, s \geq 2} b(0,1, p, q, k, s) \sum_{j=1}^{N} \widehat{v}_{p q}^{(j-1)}(\tau) \widehat{v}_{k s}^{(N-j)}(\tau)\right] d \tau \\
= & \widetilde{A}_{01} \exp \left(-\kappa_{01} t\right)+c^{N}(N+1)^{-2} O\left(\exp \left(-2 \kappa_{01} t\right)\right)
\end{aligned}
$$

where

$$
\widetilde{A}_{01}=\frac{b(0,1,0,1,0,1)}{\kappa_{01}} \widetilde{A}, \quad \widetilde{A}=\sum_{N=0}^{\infty} \varepsilon^{N+1} \sum_{j=1}^{N} A_{01}^{(j-1)} A_{01}^{(N-j)},
$$


and the series above converge absolutely and uniformly with respect to $\varepsilon \in$ $\left[0, \varepsilon_{0}\right]$ and $t \geq 0$. Recalling (1.8) and taking into account (6.3) we deduce that

$$
\left|\widehat{u}_{01}(t)-A_{01} \exp \left(-\kappa_{01} t\right)-\widetilde{A}_{01} \exp \left(-2 \kappa_{01} t\right)\right| \leq C \exp \left(-3 \kappa_{01} t\right)
$$

where

$$
A_{01}=\sum_{N=0}^{\infty} \varepsilon^{N+1} A_{01}^{(N)} .
$$

The asymptotic estimate (6.3), (6.5) permits us to single out the major parts in all $\widehat{v}_{m n}^{(N)}(t), N \geq 1$ (and consequently in $\widehat{u}_{m n}(t)$ ), which contribute to the second term of the long-time asymptotics. The functions $\widehat{v}_{m n}^{(0)}(t)$ with $m, n \geq 1$ and $m=0, n \geq 2$ do not make a contribution to this term since, according to (4.12), they contain the exponential factor $\exp \left(-\kappa_{m n} t\right)$ and $\kappa_{m n} \geq \kappa_{11}>3 \kappa_{01}$ for $m, n \geq 1 ; \kappa_{m n} \geq \kappa_{02}>3 \kappa_{01}$ for $m=0, n \leq 2$. Indeed,

$$
\kappa_{11}-3 \kappa_{01}=\lambda_{01}^{2}\left\{\nu \lambda_{01}^{2}\left[\left(\lambda_{11} / \lambda_{01}\right)^{4}-3\right]+\left[3-\left(\lambda_{11} / \lambda_{01}\right)^{2}\right]\right\}>0
$$

since $\lambda_{11} \simeq 4.493$ and $\lambda_{01}=\pi$. It is also easy to check that

$$
\kappa_{02}-3 \kappa_{01}=\lambda_{02}^{2}\left\{\nu \lambda_{02}^{2}\left[\left(\lambda_{02} / \lambda_{01}\right)^{4}-3\right]+\left[3-\left(\lambda_{02} / \lambda_{01}\right)^{2}\right]\right\}>0
$$

because $\lambda_{02} / \lambda_{01}=2$ and $\nu \lambda_{02}^{2}>1$.

For $m, n \geq 1, N \geq 1$ we can write

$$
\begin{aligned}
\widehat{v}_{m n}^{(N)}(t)= & \exp \left(-\kappa_{m n} t\right) \int_{0}^{t} \exp \left(\kappa_{m n} \tau\right)\left[b(m, n, 0,1,0,1) \sum_{j=1}^{N} \widehat{v}_{01}^{(j-1)}(\tau) \widehat{v}_{01}^{(N-j)}(\tau)\right. \\
& \left.+\sum_{p, k \geq 1 ; q, s \geq 2} b(m, n, p, q, k, s) \sum_{j=1}^{N} \widehat{v}_{p q}^{(j-1)}(\tau) \widehat{v}_{k s}^{(N-j)}(\tau)\right] d \tau
\end{aligned}
$$

By means of (6.3), (6.5) we get

$$
\begin{aligned}
\widehat{v}_{m n}^{(N)}(t)= & \exp \left(-\kappa_{m n} t\right) \int_{0}^{t} \exp \left(\kappa_{m n} \tau\right) \\
& \times\left[\exp \left(-2 \kappa_{01} \tau\right) b(m, n, 0,1,0,1) \sum_{j=1}^{N} A_{01}^{(j-1)} A_{01}^{(N-j)}\right. \\
& \left.+c^{N}(N+1)^{-2} O\left(\exp \left(-3 \kappa_{01} \tau\right)\right)\right] \\
= & \exp \left(-2 \kappa_{01} t\right) \frac{b(m, n, 0,1,0,1)}{\kappa_{m n}-2 \kappa_{01}} \sum_{j=1}^{N} A_{01}^{(j-1)} A_{01}^{(N-j)} \\
& +c^{N}(N+1)^{-2} O\left(\exp \left(-3 \kappa_{01} t\right)\right)
\end{aligned}
$$


Therefore, by (1.8), for $m, n \geq 1, t \geq 0$ we have

$$
\left|\widehat{u}_{m n}(t)-\widetilde{u}_{m n}(t)\right| \leq C \lambda_{m n}^{-5 / 2} \sqrt{m+1} \exp \left(-3 \kappa_{01} t\right),
$$

where

$$
\widetilde{u}_{m n}(t)=\widetilde{A} \frac{b(m, n, 0,1,0,1)}{\kappa_{m n}-2 \kappa_{01}} \exp \left(-2 \kappa_{01} t\right) .
$$

Recalling that

$$
j_{0}\left(\lambda_{01} r\right)=\sqrt{\frac{\pi}{2 r}} J_{1 / 2}(\pi r)=\frac{\sin (\pi r)}{\sqrt{\pi} r},
$$

setting

$$
B_{01}=\sqrt{\pi} A_{01}, \quad \widetilde{B}_{01}=\sqrt{\pi} \widetilde{A}_{01},
$$

and combining (6.1), (6.6), and (6.7) we obtain (3.2).

7. Conclusion. We have presented a method of constructing solutions of semilinear dissipative evolution equations in bounded domains. For small initial data this approach permits one not only to construct solutions in the form of eigenfunction expansion series, but also to obtain long-time asymptotic expansions. As an application we have considered the global-intime solutions of the Kuramoto-Sivashinsky in a ball in the linearly stable case $\nu>1 / \pi^{2}$. The solution is presented in the form of an expansion in eigenfunctions of the Laplace operator in the unit ball. The coefficients of the corresponding series are calculated by means of perturbation theory. The second-order long-time asymptotics calculated above is essentially nonlinear.

Acknowledgements. The author would like to thank Prof. Piotr Biler and Dr. Grzegorz Karch for useful discussions and the referee for careful reading of the manuscript.

\section{References}

[1] D. Armbruster, J. Guckenheimer and P. Holmes, Kuramoto-Sivashinsky dynamics on the center unstable manifold, SIAM J. Appl. Math. 49 (1989), 676-691.

[2] A. V. Babin and M. I. Vishik, Attractors of Evolutionary Equations, North-Holland, Amsterdam, 1992.

[3] D. J. Benney, Long waves on liquid films, J. Math. Phys. 45 (1966), 150-155.

[4] P. Collet, J. P. Eckmann, H. Epstein and J. Stubbe, A global attracting set for the Kuramoto-Sivashinsky equation, Comm. Math. Phys. 152 (1993), 203-214.

[5] C. Foiaş, B. Nicolaenko, G. R. Sell and R. Temam, Inertial manifolds for dissipative partial differential equations, C. R. Acad. Sci. Paris Sér. I 301 (1995), 199-241.

[6] -, - - - - Inertial manifolds for the Kuramoto-Sivashinsky equation and an estimate of their lowest dimension, J. Math. Pures Appl. 67 (1988), 197-226.

[7] J. Goodman, Stability of the Kuramoto-Sivashinsky equation and related systems, Comm. Pure Appl. Math. 47 (1994), 293-306. 
[8] D. Henry, Geometric Theory of Semilinear Parabolic Equations, Lecture Notes in Math. 840, Springer, Berlin, 1981.

[9] A. P. Hooper and R. Grimshaw, Nonlinear instability at the interface between two viscous fluids, Phys. Fluids 28 (1985), 37-45.

[10] E. Jahnke, F. Emde and F. Lösch, Tables of Higher Functions, 6th ed., Teubner, Stuttgart, 1960.

[11] Y. Kuramoto and T. Tsuzuki, Persistent propagation of concentration waves in dissipative media far from the thermal equilibrium, Progr. Theoret. Phys. 55 (1976), 356-369.

[12] Y. Kuramoto and T. Yamada, Turbulent state in chemical reactions, ibid. 56 (1976), 679.

[13] D. Michelson, Steady solutions of the Kuramoto-Sivashinsky equation, Phys. D 19 (1986), 89-111.

[14] - Bunsen flames as steady solutions of the Kuramoto-Sivashinsky equation, SIAM J. Math. Anal. 23 (1991), 364-386.

[15] —, Stability of the Bunsen flame profiles in the Kuramoto-Sivashinsky equation, SIAM J. Math. Anal. 27 (1996), 765-781.

[16] L. Molinet, Local dissipativity in $L_{2}$ for the Kuramoto-Sivashinsky equation in spatial dimension two, J. Dynamics Differential Equations 12 (2000), 535-556.

[17] P. I. Naumkin and I. A. Shishmarëv, Nonlinear Nonlocal Equations in the Theory of Waves, Transl. Math. Monographs 133, Amer. Math. Soc., Providence, RI, 1994.

[18] B. Nicolaenko and B. Scheurer, Remarks on the Kuramoto-Sivashinsky equation, Phys. D 12 (1984), 391-395.

[19] B. Nicolaenko, B. Scheurer and R. Temam, Some global dynamical properties of the Kuramoto-Sivashinsky equation: nonlinear stability and attractors, ibid. 16 (1985), $155-183$.

[20] A. K. Pani and H. Saranga, Finite element Galerkin method for the "good" Boussinesq equation, Nonlinear Anal. 29 (1997), 937-956.

[21] G. Raugel and G. Sell, Navier-Stokes equations on thin 3D domains. I: Global attractors and global regularity of solutions, J. Amer. Math. Soc. 6 (1993), 503-568.

[22] G. Sansone, Orthogonal Functions, Interscience Publ., New York, 1962.

[23] G. R. Sell and M. Taboada, Local dissipativity and attractors for the KuramotoSivashinsky equation in thin 2D domains, Nonlinear Anal. 18 (1992), 671-687.

[24] G. I. Sivashinsky, On flame propagation under conditions of stoichiometry, SIAM J. Appl. Math. 39 (1980), 67-82.

[25] - Instabilities, pattern formation, and turbulence in flames, Ann. Rev. Fluid Mech. 15 (1983), 179-199.

[26] K. R. Stromberg, Introduction to Classical Real Analysis, Wadsworth, Belmont, CA, 1981.

[27] E. C. Titchmarsh, Eigenfunction Expansions Associated with Second-Order Differential Equations, Clarendon Press, Oxford, 1958.

[28] G. Tolstov, Fourier Series, Dover Publ., New York, 1962.

[29] J. Topper and T. Kawahara, Approximate equations for nonlinear waves on a viscous fluid, J. Phys. Soc. Japan 44 (1978), 663-666.

[30] V. V. Varlamov, On spatially periodic solutions of the damped Boussinesq equation, Differential Integral Equations 10 (1997), 1197-1211.

[31] —, On the initial-boundary value problem for the damped Boussinesq equation, Discrete Continuous Dynamical Systems 4 (1998), 431-444.

[32] -, Long-time asymptotics of solutions of the second initial-boundary value problem for the damped Boussinesq equation, Abstract Appl. Anal. 2 (1998), 97-115. 
[33] V. V. Varlamov, On the damped Boussinesq equation in a circle, Nonlinear Anal. 38 (1999), 447-470.

[34] - On the spatially two-dimensional Boussinesq equation in a circular domain, ibid., to appear.

[35] - , Nonlinear heat equation with a fractional Laplacian in a disk, Colloq. Math. 81 (1999), 101-122.

[36] - Long-time asymptotics for the nonlinear heat equation with a fractional Laplacian in a ball, Studia Math. 142 (2000), 71-99.

[37] M. Vishik, Asymptotic Behavior of Solutions of Evolutionary Equations, Cambridge Univ. Press, Cambridge, 1992.

[38] G. N. Watson, A Treatise on the Theory of Bessel Functions, 2nd ed., Cambridge Univ. Press, London, 1966.

[39] C. E. Wayne, Invariant manifolds for parabolic partial differential equations on unbounded domains, Arch. Rational Mech. Anal. 138 (1997), 279-306.

Department of Mathematics

The University of Texas at Austin

Austin, TX 78712-1082, U.S.A.

E-mail: varlamov@math.utexas.edu 